\title{
Knockdown of ADAM10 inhibits migration and invasion of fibroblast-like synoviocytes in rheumatoid arthritis
}

\author{
DAN LI $^{1}$, ZHITAO XIAO ${ }^{2}$, GANG WANG $^{3}$ and XIANJI SONG ${ }^{3}$ \\ ${ }^{1}$ Department of Radiology, The First Teaching Hospital of Jilin University; ${ }^{2}$ Department of \\ Engineering Mechanics, Jilin University, Changchun, Jilin 130021; ${ }^{3}$ Department of Orthopaedics, \\ The Third Teaching Hospital of Jilin University, Changchun, Jilin 130033, P.R. China
}

Received September 19, 2014; Accepted June 5, 2015

DOI: $10.3892 / \mathrm{mmr} .2015 .4011$

\begin{abstract}
Rheumatoid arthritis (RA) is a systemic autoimmune disease with high rates of morbidity and mortality. Previous studies proposed that the A disintegrin and metalloprotease (ADAM) family is involved in the regulation of inflammation and arthritis. Thus, the present study investigated whether ADAM10 is involved in the progression of RA. The effects of ADAM10 small interfering (si)RNA on the expression levels of tumor necrosis factor (TNF)- $\alpha$, interleukin (IL)-6, IL-8 and chemokine (C-X-C motif) ligand 16 (CXCL16) were determined in fibroblast-like synoviocytes (FLS). In addition, the effects of ADAM10 siRNA on cell proliferation, invasion and migration in human RA-FLS were assessed in vitro. The therapeutic efficacy and side-effects of ADAM10 siRNA were examined in a mouse model of collagen-induced arthritis (CIA). In vitro, ADAM10 silencing suppressed the expression of TNF- $\alpha$, IL-6, IL-8 and CXCL16 in lipopolysaccharide(LPS)-stimulated human RA-FLS. LPS-induced RA-FLS proliferation, migration and invasion were significantly attenuated by ADAM10 knockdown. ADAM10 silencing inhibited the secretion of vascular endothelial growth factor A (VEGF-A) and matrix metalloproteinase (MMP)-3 and -9 from LPS-stimulated human RA-FLS, in addition to inhibiting the phosphoinositide 3-kinase/AKT activation in LPS-stimulated human RA-FLS. In vivo, treatment with siRNA against ADAM10 for three weeks reduced the arthritis score. Serum levels of VEGF-A, MMP-3 and MMP-9 were also reduced in CIA mice. These observations indicate that the inhibition of ADAM10 may be a viable therapeutic target in the
\end{abstract}

Correspondence to: Professor Gang Wang, Department of Orthopaedics, The Third Teaching Hospital of Jilin University, 126 Xiantai Street, Changchun, Jilin 130033, P.R. China

E-mail: wanggangletter@tom.com

Key words: ADAM10, rheumatoid arthritis, rheumatoid arthritis fibroblast-like synoviocytes, migration, invasion amelioration of disease progression in RA by attenuating FLS proliferation, migration and invasion.

\section{Introduction}

Rheumatoid arthritis (RA) is a chronic inflammatory autoimmune disease, characterized by excessive synovial hyperplasia, infiltration of inflammatory mononuclear cells and formation of pannus over a joint surface, leading to tissue destruction and functional disability (1,2). Multiple cell types, including macrophages, osteoclasts and chondrocytes are present in the damaged joints of RA $(3,4)$. However, growing evidence indicates that activated fibroblast-like synoviocytes (FLS), which are highly present in the RA synovium, serve key roles in disease progression by producing proinflammatory cytokines and proteases that facilitate cartilage destruction $(2,5,6)$. It has been demonstrated that activated RA-FLS migrate into and invade the cartilage and bone, participating in the formation of synovial pannus and joint destruction $(5,7,8)$. Thus, novel therapeutic strategies with the ability to modulate activated FLS migration and invasion are required to prevent and inhibit the progressive destruction resulting from RA.

The members of the A disintegrin and metalloproteinase (ADAM) family are proteases that are responsible for the liberation of a variety of cell surface-expressed proteins (9). It has been demonstrated that ADAMs are involved in various inflammatory and degenerative pathological conditions (9). ADAM10, a member of the ADAM family, is involved in the shedding of numerous substrates, which are key in cancer progression, allergic responses and inflammatory disease (10-13). In addition, ADAM-10 has been reported to cleave various inflammatory and angiogenic mediators from the cell surface, such as chemokine (C-X-C motif) ligand 16 (CXCL16) (14) and fractalkine (15). Notably, a previous study indicated that ADAM-10 was overexpressed in RA synovial tissue and was critical in angiogenesis in RA (16). However, the detailed role of ADAM-10 in RA remains to be fully elucidated. Therefore, the aim of the present study was to analyze the association between ADAM10 expression and the expression of proinflammatory cytokines in mouse FLS. The effects of ADAM10 small interfering RNA (siRNA) in a mouse model of collagen-induced arthritis (CIA) were 
investigated, in addition to observations of cell proliferation, migration and invasion in vitro.

\section{Materials and methods}

Patients and isolation of FLS. Written informed consent was obtained from individual patients and the experimental protocol was approved by the Medical Ethics Committee of the Third Teaching Hospital of Jilin University (Changchun, China). RA-FLS were obtained from three patients with RA who underwent a synovectomy. RA-FLS were isolated from synovial tissues by enzymatic digestion, as previously described (17). FLS were grown in Dulbecco's modified Eagle's medium (Gibco Life Technologies, Carlsbad, CA, USA) containing $10 \%$ fetal bovine serum (FBS; Invitrogen Life Technologies, Carlsbad, CA, USA), supplemented with $100 \mathrm{mg} / \mathrm{ml}$ streptomycin and $100 \mathrm{U} / \mathrm{ml}$ penicillin (Sigma-Aldrich, St. Louis, MO, USA) in a humidified incubator at $37^{\circ} \mathrm{C}$ under $5 \% \mathrm{CO}_{2}$. RA-FLS cells used for the experiments were at the third to sixth passage.

FLS transfection. The control and ADAM10-specific siRNA were obtained from Shanghai GenePharma (Shanghai, China). When RA-FLS reached 70-90\% confluence, the cells were transfected with ADAM10 siRNA or control siRNA using Lipofectamine 2000 reagent (Invitrogen Life Technologies) according to the manufacturer's instructions. The efficacy of ADAM10 silencing was determined by western blot analysis.

Measurement of tumor necrosis factor (TNF)- $\alpha$, interleukin (IL)-6, IL-8 and CXCL16 production. In order to measure TNF- $\alpha$, IL-6, IL- 8 and CXCL16 production, the cells were pretreated with ADAM10 siRNA for $8 \mathrm{~h}$ followed by stimulation with $10 \mu \mathrm{g} / \mathrm{ml}$ lipopolysaccharide (LPS; Sigma-Aldrich) for $24 \mathrm{~h}$. The culture supernatants were harvested by centrifugation at $1,000 \mathrm{x}$ for $5 \mathrm{~min}$ at room temperature, $24 \mathrm{~h}$ subsequent to LPS stimulation, and the concentrations of TNF- $\alpha$, IL-6, IL- 8 and CXCL16 were measured using ELISA kits for human TNF- $\alpha$, IL-6, IL-15 and CXCL16 (Bio-Techne, Minneapolis, MN, USA) according to the manufacturer's instructions. The concentration of each was normalized relative to the total number of cells.

Cell proliferation. To measure the effect of ADAM10 siRNA on cell proliferation, the 5-bromo-2-deoxyuridine (BrdU) assay (Merck Millipore, Darmstadt, Germany) was conducted. Briefly, RA-LFS were transfected with ADAM10 siRNA and control SiRNA for $24 \mathrm{~h}$, followed by stimulation with $10 \mu \mathrm{g} / \mathrm{ml}$ LPS for $48 \mathrm{~h}$. Subsequently, cell proliferation was detected by the BrdU Cell Proliferation assay kit according to the manufacturer's instructions.

Cell migration and invasion assay. To assess the effect of ADAM10 siRNA on cell migration and invasion using Transwell insert chambers (Corning Incorporated, New York, NY, USA). For the migration assay, RA-LFS were transfected with ADAM10 siRNA and control siRNA for $24 \mathrm{~h}$, followed by stimulation with $10 \mu \mathrm{g} / \mathrm{ml}$ LPS for $48 \mathrm{~h}$. The cells $\left(1 \times 10^{5}\right)$ were then plated in the upper chamber in serum-free medium, in triplicate. Medium containing 20\% FBS in the lower chamber served as the chemoattractant. Subsequent to culture for $24 \mathrm{~h}$, the media was removed from the upper chamber by wiping with a cotton swab and the cells that had migrated to the lower surface of the filter were fixed in $70 \%$ ethanol for $30 \mathrm{~min}$ and stained with $0.2 \%$ crystal violet (Sigma-Aldrich) for $10 \mathrm{~min}$. Cell migration was measured by counting five randomly selected fields per filter under a light microscope (Olympus, Tokyo, Japan).

For the invasion assay, $3 \times 10^{5}$ cells were seeded into upper chambers pre-coated with Matrigel (BD Biosciences, Franklin Lakes, NJ, USA) in serum-free medium, in triplicate, and the subsequent steps were the same as those of the aforementioned migration assay up until the cells were stained with crystal violet. The number of cells invading the Matrigel was measured in five randomly selected fields using an inverted microscope (X51; Olympus, Tokyo, Japan).

Western blot analysis. Cells were lysed by incubation on ice for $30 \mathrm{~min}$ in lysis buffer containing the Complete Protease Inhibitor Cocktail (Roche Diagnostics GmbH, Mannheim, Germany). Equal quantities of protein (15 $\mu \mathrm{g} / \mathrm{lane})$ from the cell lysates were separated on an 8-15\% SDS-PAGE (Invitrogen Life Technologies) and transferred onto nitrocellulose membranes (Santa Cruz Biotechnology, Inc., Santa Cruz, CA, USA). The membrane was incubated for $2 \mathrm{~h}$ in phosphate-buffered saline (PBS; Sigma-Aldrich) plus $0.1 \%$ Tween-20 (Sigma-Aldrich) and 5\% nonfat milk to block non-specific binding. The membranes were then incubated with the following mouse monoclonal primary antibodies: Anti-ADAM10 (1:1,000; Santa Cruz Biotechnology, Inc.; cat. no. sc-28358), anti-phosphorylated (p)-AKT (Ser473; 1:1,000; cat. no. 4000), anti-AKT (1:3,000; cat. no. 2920), anti-p-phosphoinositide 3-kinase (p-PI3K) (Tyr458; 1:1,000; cat. no. 4228), anti-PI3K (1:5,000; cat. no. 4249) and anti- $\beta$-actin (1:8,000; cat. no. 3700 ; all from Cell Signaling Technology, Inc., Danvers, MA, USA) at room temperature for 2 h. Following washing with PBS, the anti-mouse secondary horseradish peroxidase-conjugated antibody (1:10,000; GE Healthcare Life Sciences, Uppsala, Sweden) was added for $2 \mathrm{~h}$. Protein bands were visualized with enhanced chemiluminescence reagent (GE Healthcare Life Sciences, Velizy-Villacoublay, France).

Initiation of CIA and treatment of CIA with ADAMIO siRNA in vivo. Thirty male DBA/1 mice (age, 6-8 weeks; weight, 200-250 g) were purchased from the Institute of Laboratory Animal Science of Jilin University (Changchun, China). All animal experiments were performed in accordance with the National Institutes of Health Guide for the Care and Use of Laboratory Animals and with the approval of the Institutional Animal Ethics Committee of Jilin University. Mice were immunized on day zero and boosted on day seven with an intradermal injection of bovine type II collagen in Freund's adjuvant (Sigma-Aldrich) as described previously (17).

Complexes of siRNA and atelocollagen [Boppard (Beijing) Co., Ltd., Beijing, China] were prepared as previously described (18). Subsequently, siRNA/atelocollagen complexes $(0.5 \mathrm{mg} / \mathrm{kg}$ body weight $)$ were administered to the CIA mice twice per week for 3 weeks, as previously described (19). 
A

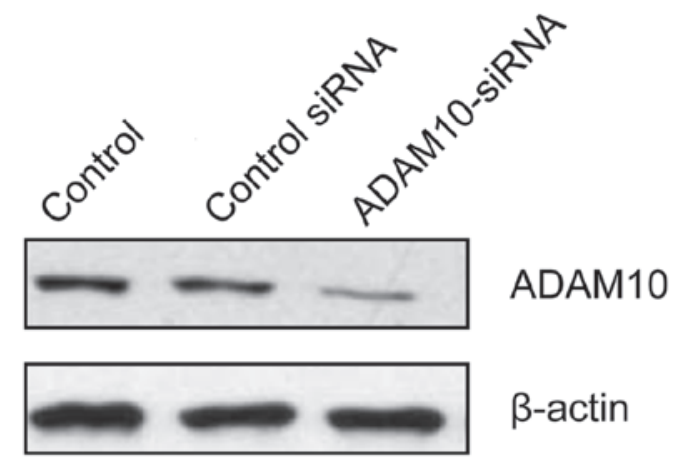

B

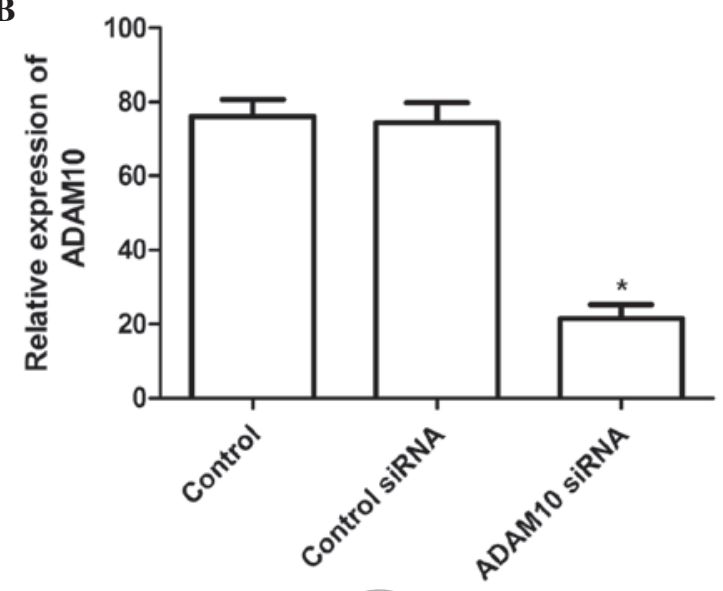

Figure 1. Silencing ADAM10 suppresses ADAM10 expression of human rheumatoid arthritis fibroblast-like synoviocytes. (A) Western blot analysis of ADAM10 protein expression $48 \mathrm{~h}$ subsequent to transfection with ADAM10 siRNA or control siRNA. (B) Quantitative analysis of the relative levels of ADAM10 expression. The data are expressed as the mean \pm standard deviation (" $\mathrm{P}<0.01$ vs. control siRNA). ADAM10, A disintegrin and metalloprotease 10; siRNA, small interfering RNA.

Arthritic score. Clinical arthritic scoring was performed every three days according to the following scoring system $(19,20)$ : 0 , Normal; 1 , mild, but definite swelling of either the ankle or digits; 2 , moderate redness and swelling of an ankle \pm any number of digits; 3 , maximal redness and swelling of the entire paw and digits with or without ankylosis. The maximum score per paw was 3 with a total score of 12 per mouse.

Measurement of vascular endothelial growth factor $A$ (VEGF-A), matrix metalloproteinase (MMP)-3 and MMP-9 levels in vitro and in vivo. For the measurement of VEGF-A, MMP-3 and MMP-9 levels in the supernatants, RA-FLS cells were seeded at a density of $2 \times 10^{6}$ cells $/ \mathrm{ml}$ and pretreated with ADAM10 siRNA for $24 \mathrm{~h}$ followed by stimulation with LPS for $48 \mathrm{~h}$. Cell supernatants were centrifuged at 1,000 x g for $5 \mathrm{~min}$ at room temperature to remove any cell debris prior to ELISA analysis. The mice were sacrificed by cervical dislocation upon completion of the experiment and ELISA was performed to determine the levels of VEGF-A, MMP-3 and MMP-9, using specific ELISA kits (R\&D Systems China Co., Ltd., Shanghai, China) according to the manufacturer's instructions.

Statistical analysis. Data are expressed as the mean \pm standard deviation. The differences between groups were analyzed by one-way analysis of variance and post hoc analysis with Dunnett's multiple comparison test and Student's t-tests using GraphPad Prism version 5.01 (GraphPad Software, Inc., La Jolla, CA, USA). P $<0.05$ was considered to indicate a statistically significant difference.

\section{Results}

Knockdown of ADAM10 inhibits ADAM10 expression in $R A-F L S$. To evaluate the silencing capacity of ADAM10, RA-FLS cells were transfected with ADAM10 siRNA and control siRNA, and the effect of ADAM10 silencing was characterized by western blot analysis two days post-transfection. As presented in Fig. 1, transfection with control siRNA did not modulate the levels of ADAM10 expression in RA-FLS cells, as compared with control RA-FLS cells. By contrast, transfection with ADAM10 siRNA significantly reduced the levels of ADAM10 expression in RA-FLS cells compared with control RA-FLS cells $(\mathrm{P}<0.01)$.

ADAM10 siRNA inhibits LPS-induced TNF- $\alpha, I L-6, I L-8$ and CXCL16 expression in RA-FLS. To quantify TNF- $\alpha$, IL-6, IL- 8 and CXCL16 production, RA-FLS were pretreated with ADAM10 siRNA for $24 \mathrm{~h}$ followed by stimulation with human LPS for $48 \mathrm{~h}$, then ELISA assays were performed. The results indicate that transfection of ADAM10 siRNA significantly reduces the production of TNF- $\alpha$, IL- 6 , IL-8 and CXCL16 in human LPS-induced RA-FLS cells, when compared with cells transfected with control siRNA (Fig. 2; $\mathrm{P}<0.05)$.

ADAM10 siRNA inhibits LPS-induced RA-FLS proliferation, migration and invasion of cells. RA-FLS were pretreated with ADAM10 siRNA for $24 \mathrm{~h}$ followed by stimulation with human LPS for $48 \mathrm{~h}$. Subsequently, the impact of ADAM10 silencing on the proliferation, migration and invasion of RA-FLS was characterized by BrdU assays, and Transwell migration and invasion assays, respectively. BrdU assays indicated that ADAM10 siRNA significantly repressed LPS-induced RA-FLS proliferation (Fig. 3A; $\mathrm{P}<0.01$ ). In addition, it was demonstrated that the numbers of migrated ADAM10 siRNA RA-FLS were significantly reduced when compared with the control cells (Fig. 3B; $\mathrm{P}<0.01$ ). A similar pattern in the numbers of invaded cells was observed in the different groups of RA-FLS (Fig. 3C; P<0.01). Hence, knockdown of ADAM10 expression appeared to inhibit the proliferation, migration and invasion of LPS-induced RA-FLS in vitro.

ADAM10 siRNA inhibits the activation of the PIBK/AKT pathway in RA-FLS cells. To elucidate the underlying mechanisms of the action of ADAM10 silencing in inhibiting the proliferation, migration and invasion of human RA-FLS cells, the impact of ADAM10 silencing on the PI3K/AKT pathway 
A

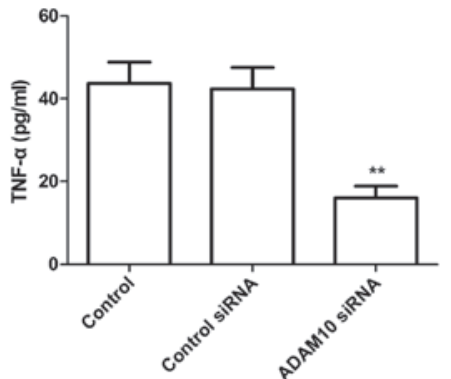

$\mathbf{C}$

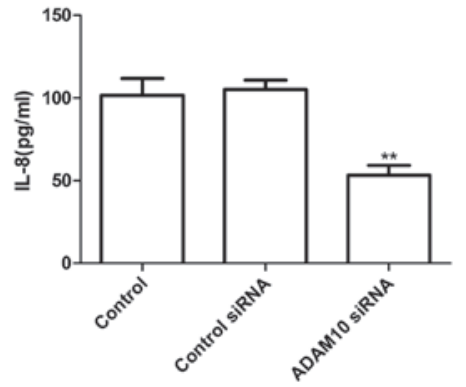

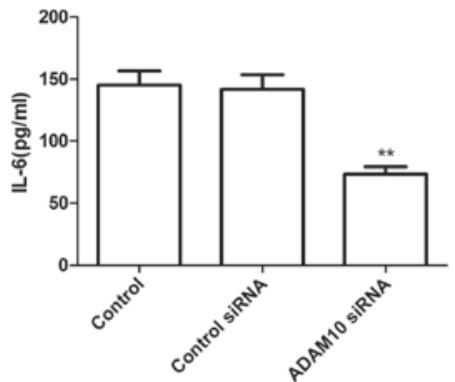

D

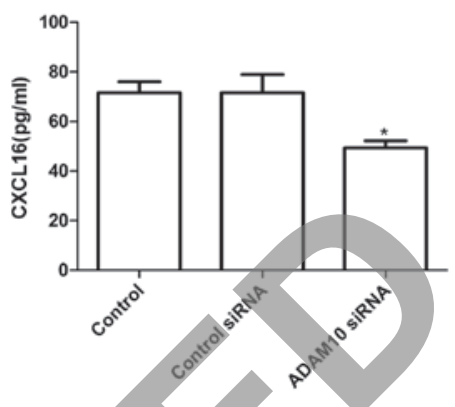

Figure 2. Silencing ADAM10 inhibited TNF- $\alpha$, IL-6, IL-8 and CXCL16 expression in human LPS-induced RA-FLS, which were transfected with ADAM10 siRNA or control siRNA and stimulated with $10 \mathrm{mg} / \mathrm{ml}$ LPS. Culture supernatants were collected $24 \mathrm{~h}$ later and concentrations of (A) TNF- $\alpha$, (B) IL-6, (C) IL-8 and (D) CXCL16 were determined by ELISA. ${ }^{*} \mathrm{P}<0.05,{ }^{* *} \mathrm{P}<0.01$ vs. control siRNA. ADAM10, A disintegrin and metalloprotease 10; TNF- $\alpha$, tumor necrosis factor- $\alpha$; IL, interleukin; LPS, lipopolysaccharide; RA-FLS, rheumatoid arthritis fibroblast-like synoviocytes; siRNA, small interfering RNA; CXCL16, chemokine (C-X-C motif) ligand 16.
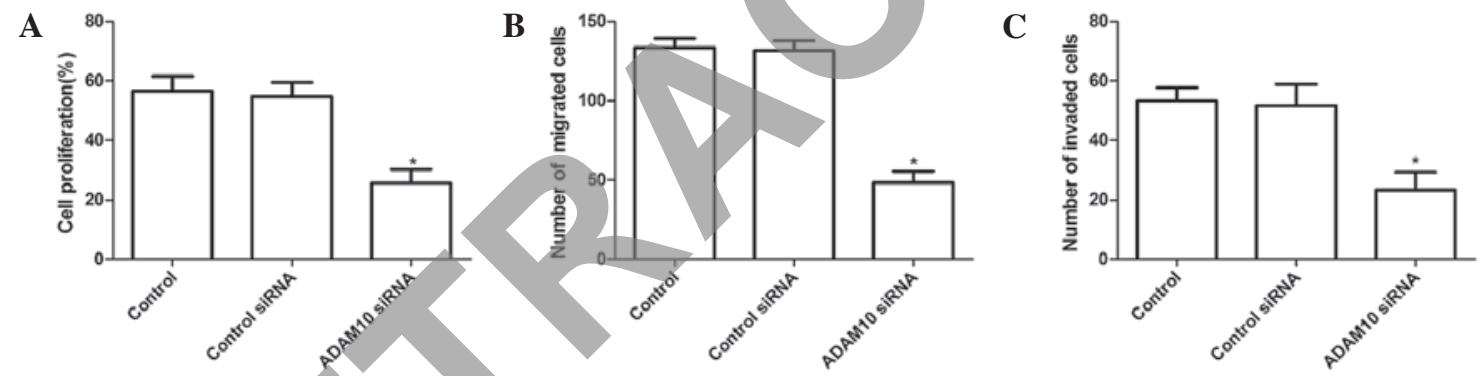

Figure 3. Silencing ADAM10 inhibited LPS-induced cell proliferation, migration and invasion. Rheumatoid arthritis fibroblast-like synoviocytes were pretreated with ADAM10 siRNA for $24 \mathrm{~h}$ and exposed to human LPS for $48 \mathrm{~h}$. (A) Cellular proliferation was measured using the 5-bromo-2-deoxyuridine incorporation assay. (B) The number of migrated cells was determined using the Transwell assay. (C) The number of invaded cells was determined using the Matrigel assay. "P<0.01 vs. control siRNA. ADAM10, A disintegyin and metalloprotease 10; LPS, lipopolysaccharide; siRNA, small interfering RNA.

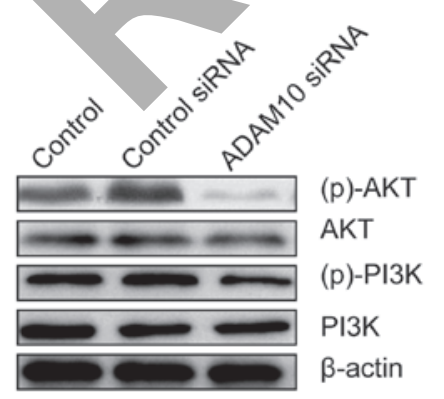

Figure 4. Silencing ADAM10 inhibits the PI3K and AKT phosphorylation in LPS-induced RA-FLS. RA-FLS were pretreated with ADAM10 siRNA for $24 \mathrm{~h}$ and exposed to human LPS for $48 \mathrm{~h}$. Western blotting was performed with specific antibodies against the indicated proteins. $\beta$-actin served as an internal control. ADAM10, A disintegrin and metalloprotease 10; , phosphorylated; PI3K, phosphoinositide 3-kinase; siRNA, small interfering RNA; RA-FLS, rheumatoid arthritis fibroblast-like synoviocytes.

in RA-FLS was evaluated. As presented in Fig. 4, compared with control RA-FLS and RA-FLS transfected with control
siRNA, silencing ADAM10 resulted in a significant suppression of the phosphorylation of PI3K and AKT in LPS-induced RA-FLS cells, without alteration of the total PI3K and AKT protein levels in the different groups. Thus indicating that knockdown of ADAM10 expression inhibits the PI3K/AKT pathway in human RA-FLS.

ADAM10 siRNA reduced the arthritis score in CIA mice. The effect of silencing ADAM10 on the arthritis score in the CIA mouse model was investigated. The arthritis score was evaluated every three days. Treatment with ADAM10 siRNA was initiated on day 22 following the first immunization, when clear onset of arthritis had occurred. It was observed that silencing of ADAM10 reduced the arthritis score of CIA in mice during the treatment (Fig. 5; $\mathrm{P}<0.05$ ).

ADAMIO siRNA inhibited the expression of VEGF-A, $M M P-3$, and MMP-9 in vitro and in vivo. Invasion-associated proteins including VEGF-A, MMP-3 and MMP-9 were investigated in human RA-FLS. The cells were pretreated 


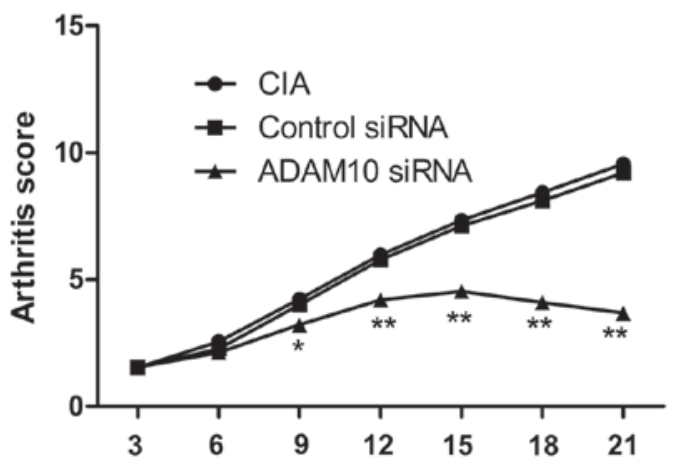

Figure 5. Silencing ADAM10 reduced the arthritis score in CIA mice. The arthritis score was evaluated every three days. Treatment with ADAM10 siRNA was initiated on day 22 following the first immunization, when clear onset of arthritis had occurred. ${ }^{*} \mathrm{P}<0.05,{ }^{* *} \mathrm{P}<0.01$ vs. CIA rats. ADAM10, A disintegrin and metalloprotease 10; CIA, collagen-induced arthritis; siRNA, small interfering RNA.

A
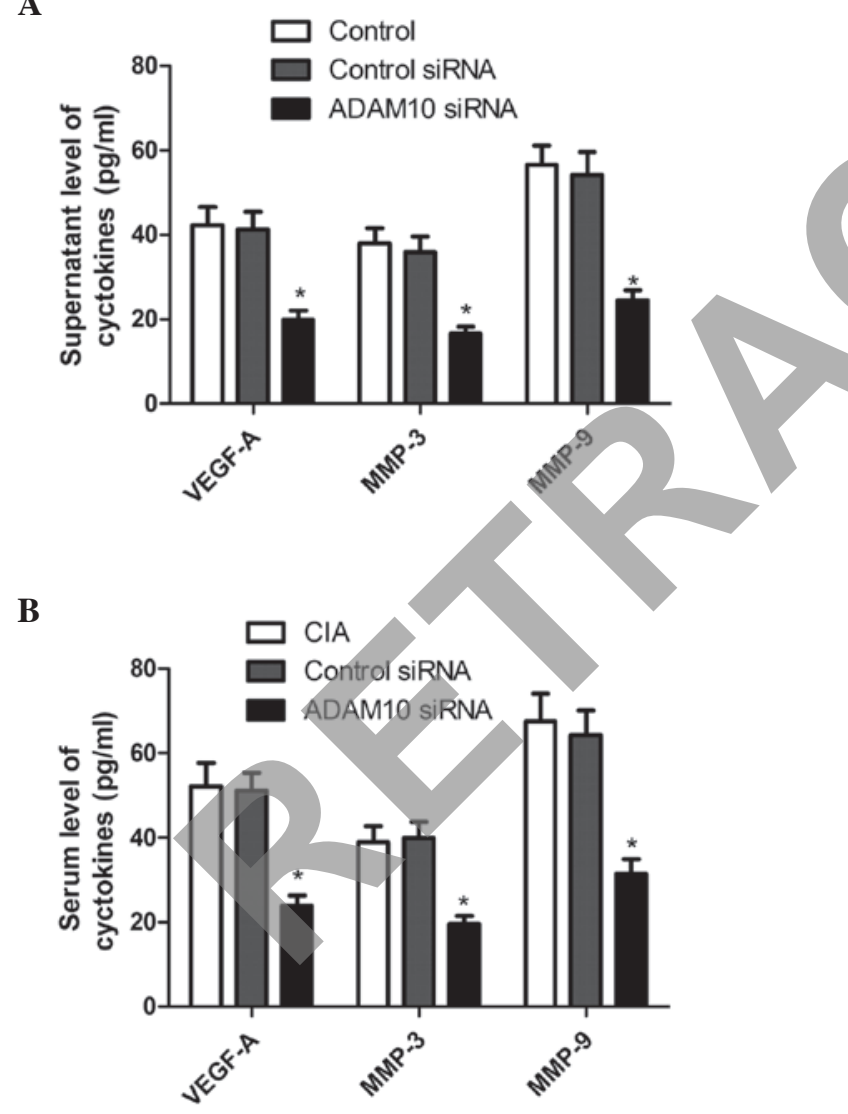

Figure 6. Silencing ADAM10 inhibited the expression of VEGF-A, MMP-3 and MMP-9 in vitro and in vivo. (A) Rheumatoid arthritis fibroblast-like synoviocytes were pretreated with ADAM10 siRNA for $24 \mathrm{~h}$ and exposed to human LPS for $48 \mathrm{~h}$. VEGF-A, MMP-3 and MMP-9 were measured using the ELISA at a wavelength of $490 \mathrm{~nm}$. (B) Serum levels of VEGF-A, MMP-3 and MMP-9 were measured using the ELISA assay at a wavelength of $490 \mathrm{~nm}$. ${ }^{*} \mathrm{P}<0.01$ vs. control siRNA. ADAM10, A disintegrin and metalloprotease 10 ; siRNA, small interfering RNA; VEGF-A, vascular endothelial growth factor A; MMP, matrix metalloproteinase; LPS, lipopolysaccharide.

with ADAM10 siRNA for $24 \mathrm{~h}$ followed by stimulation with human LPS for $48 \mathrm{~h}$. The collected supernatants were then assayed for VEGF-A, MMP-3 and MMP-9. Results of ELISA demonstrated that ADAM10 silencing substantially reduced the expression levels of VEGF-A, MMP-3 and MMP-9 in the supernatants when compared with those of the controls (Fig. 6A; $\mathrm{P}<0.01$ ).

In addition, the production of VEGF-A, MMP-3 and MMP-9 in the serum of CIA model mice was investigated. It was identified that ADAM10 siRNA inhibits the production of serum VEGF-A, MMP-3 and MMP-9, when compared with control-treated mice (Fig. 6B; $\mathrm{P}<0.01$ ).

\section{Discussion}

It is widely accepted that RA-FLS secrete multiple cytokines and growth factors (such as VEGF) that contribute to the activation of an autocrine loop, resulting in further FLS hyperplasia (21). It has also been demonstrated that RA-FLS migrate and invade into the cartilage and bone, leading to pannus formation and tissue damage during the pathogenic process of RA (22-24). Thus, it is important to identify factors that regulate the migration and invasion of RA-FLS. In the present study, the results demonstrated that transfection with ADAM10 siRNA effectively reduced the levels of ADAM10 expression in human RA-FLS. Furthermore, the results indicated that knockdown of ADAM10 expression inhibited the proliferation, migration and invasion of RA-FLS. In addition, it was observed that knockdown of ADAM10 in RA-FLS inhibited the VEGF-A, MMP-3 and MMP-9 expression levels and the activation of PI3K/AKT signaling. These data imply that ADAM10 may positively regulate the migration and invasion of human RA-FLS. Hence, ADAM10 may serve as a novel therapeutic target for treatment of RA.

Cytokines have been reported to be involved with the progression of RA and perform pathogenic roles in the establishment of rheumatoid synovitis $(24,25)$. TNF- $\alpha$ is a key inflammatory cytokine involved in the pathogenesis of RA, and inhibition of TNF- $\alpha$ expression by antagonism or the use of therapeutic agents is an effective treatment for RA $(26,27)$. IL-6 is present at high concentrations in the serum and synovial fluid of patients with RA $(28,29)$, and serves a key role in the pathogenesis of RA, including osteoporosis and an increased concentration of IL-6 in the joints around the body (28). Increasing evidence indicates that the ADAM family is involved in the regulation of inflammatory responses, and that members of this family may serve as novel therapeutic targets for the treatment of inflammatory disorders, including RA (30). Therefore, the aim of the present study was to determine whether the expression of ADAM10 was associated with inflammatory conditions in human RA-FLS. The results of the current study demonstrate that downregulation of ADAM10 expression inhibits the expression of TNF- $\alpha$, IL-6, IL- 8 and CXCL16 in LPS-induced RA-FLS cells. Therefore, ADAM10 may have potential for use as a therapeutic tool in the treatment of patients with RA who are resistant to anti-cytokine therapeutic strategies.

ADAM10, a member of the ADAM family, has been demonstrated to be involved in numerous cell processes, including proliferation, differentiation, migration and invasion $(31,32)$. A previous study identified that ADAM10 is important in modulating the chemosensitivity of hepatocellular carcinoma cells, which involves activation of the PI3K/AKT signaling 
pathway (33). Accumulating evidence demonstrates that the PI3K/AKT signaling pathway positively regulates the migration and invasion of various types of cells $(34,35)$. The results of the current study demonstrate that knockdown of ADAM10 inhibits PI3K and AKT phosphorylation, and attenuates spontaneous migration and invasion, as well as VEGF-A, MMP-3 and MMP-9 expression in human RA-FLS. MMPs have been reported to be involved in the degradation of extracellular matrix components and significant contributors to joint destruction during the process of RA (35-37). Therefore, it was hypothesized that therapeutic targeting of ADAM10 may result in the suppression of migration and invasion of human RA-FLS via the inhibition of PI3K/AKT activation, MMP-2 and MMP-9 expression.

In conclusion, the data from the present study demonstrate that knockdown of ADAM10 inhibits the production of inflammatory cytokines, the activation of PI3K/AKT signaling, and the expression of VEGF-A, MMP-3 and MMP-9 in LPS-induced RA-FLS, as well as inhibiting human RA-FLS proliferation, migration and invasion in vitro. In addition, knockdown of ADAM10 was found to reduce the arthritis score and serum levels of VEGF-A, MMP-3 and MMP-9 in vivo. These observations present novel evidence that ADAM10 may serve as a novel target for treatment of RA.

\section{References}

1. Firestein GS: Evolving concepts of rheumatoid arthritis Nature 423: 356-361, 2003.

2. Miossec P: Rheumatoid arthritis: Still a chronic disease Lancet 381: 884-886, 2013.

3. Bottini N and Firestein GS: Duality of fibroblast-like synoviocytes in RA: Passive responders and imprinted aggressors. Nat Rev Rheumatol 9: 24-33, 2013.

4. Li F, Li X, Kou L, Li Y, Meng F and Ma F: SUMO-conjugating enzyme UBC9 promotes proliferation and migration of fibroblast-like synoviocytes in rheumatoid arthritis. Inflammation 37: 1134-1141, 2014.

5. Juarez M, Filer A and Buckley CD: Fibroblasts as therapeutic targets in rheumatoid arthritis and cancer. Swiss Med Wkly 142 w13529, 2012

6. Bartok B and Firestein GS: Fibroblast-like synoviocytes: Key effector cells in rheumatoid arthritis. Immunol Rev 233: 233-255, 2010.

7. Muller-Ladner U, Kriegsmann J, Franklin BN, Matsumoto S, Geiler T, Gay RE and Gay S: Synovial fibroblasts of patients with rheumatoid arthritis attach to and invade normal human cartilage when engrafted into SCID mice. Am J Pathol 149: 1607-1615, 1996.

8. Huber LC, Distler O, Tarner I, Gay RE, Gay S and Pap T: Synovial fibroblasts: Key players in rheumatoid arthritis. Rheumatology (Oxford) 45: 669-675, 2006.

9. Pruessmeyer J and Ludwig A: The good, the bad and the ugly substrates for ADAM10 and ADAM17 in brain pathology, inflammation and cancer. Semin Cell Dev Biol 20: 164-174, 2009.

10. Schulz B, Pruessmeyer J, Maretzky T, Ludwig A, Blobel CP, Saftig P and Reiss K: ADAM10 regulates endothelial permeability and T-Cell transmigration by proteolysis of vascular endothelial cadherin. Circ Res 102: 1192-1201, 2008.

11. Eichenauer DA, Simhadri VL, von Strandmann EP, Ludwig A, Matthews V, Reiners KS, von Tresckow B, Saftig P, Rose-John S, Engert A, et al: ADAM10 inhibition of human CD30 shedding increases specificity of targeted immunotherapy in vitro. Cancer Res 67: 332-338, 2007.

12. Weskamp G, Ford JW, Sturgill J, Martin S, Docherty AJ, Swendeman S, Broadway N, Hartmann D, Saftig P, Umland S, et al: ADAM10 is a principal 'sheddase' of the low-affinity immunoglobulin E receptor CD23. Nat Immunol 7: 1293-1298, 2006.
13. McCulloch DR, Akl P, Samaratunga H, Herington AC and Odorico DM: Expression of the disintegrin metalloprotease, ADAM-10, in prostate cancer and its regulation by dihydrotestosterone, insulin-like growth factor I and epidermal growth factor in the prostate cancer cell model LNCaP. Clin Cancer Res 10: 314-323, 2004.

14. Gough PJ, Garton KJ, Wille PT, Rychlewski M, Dempsey PJ and Raines EW: A disintegrin and metalloproteinase 10-mediated cleavage and shedding regulates the cell surface expression of CXC chemokine ligand 16. J Immunol 172: 3678-3685, 2004

15. Hundhausen C, Misztela D, Berkhout TA, Broadway N, Saftig P, Reiss K, Hartmann D, Fahrenholz F, Postina R and Matthews V: The disintegrin-like metalloproteinase ADAM10 is involved in constitutive cleavage of CX3CL1 (fractalkine) and regulates CX3CL1-mediated cell-cell adhesion. Blood 102: 1186-1195, 2003.

16. Isozaki T, Rabquer BJ, Ruth JH, Haines GK III and Koch AE: ADAM-10 is overexpressed in rheumatoid arthritis synovial tissue and mediates angiogenesis. Arthritis Rheum 65: 98-108, 2013.

17. Chen SY, Wu CL, Lai MD, Lin CC, Yo YT, Jou IM, Lee CH, Weng CT, Shiau AL and Wang CR: Amelioration of rat collagen-induced arthritis through CD4+T cells apoptosis and synovial interleukin-17 reduction by indoleamine 2,3-dioxygenase gene therapy. Hum Gene Ther 22: 145-154, 2011.

18. Minakuchi Y, Takeshita F, Kosaka N, Sasaki H, Yamamoto Y, Kouno M, Honma K, Nagahara S, Hanai K, Sano A, et al: Atelocollagen-mediated synthetic small interfering RNA delivery for effective gene silencing in vitro and in vivo. Nucleic Acids Res 32: e109, 2004

19. Li F, Li X, Kou L, Li Y, Meng F and Ma F: SUMO-conjugating enzyme UBC9 promotes proliferation and migration of fibroblast-like synoviocytes in rheumatoid arthritis. Inflammation 37: 1134-1141, 2014

20. Tarrant TK, Liu P, Rampersad RR, Esserman D, Rothlein LR, Timoshchenko RG, McGinnis MW, Fitzhugh DJ, Patel DD and Fong AM: Decreased Th17 and antigen-specific humoral responses in CX3 CR1-deficient mice in the collagen-induced arthritis model. Arthritis Rheum 64: 1379-1387, 2012.

21. Afuwape AO, Kiriakidis S and Paleolog EM: The role of the angiogenic molecule VEGF in the pathogenesis of rheumatoid arthritis. Histol Histopathol 17: 961-972, 2002.

22. Muller-Ladner U, Pap T, Gay RE, Neidhart M and Gay S: Mechanisms of disease: The molecular and cellular basis of joint destruction in rheumatoid arthritis. Nat Clin Pract Rheumatol 1: 102-110, 2005.

23. Huber LC, Distler O, Tarner I, Gay RE, Gay S and Pap T: Synovial fibroblasts: Key players in rheumatoid arthritis. Rheumatology (Oxford) 45: 669-675, 2006.

24. Buchan G, Barrett K, Turner M, Chantry D, Maini RN and Feldmann M: Interleukin-1 and tumour necrosis factor mRNA expression in rheumatoid arthritis: Prolonged production of IL-1 alpha. Clin Exp Immunol 73: 449-455, 1988.

25. Choy EH and Panayi GS: Cytokine pathways and joint inflammation in rheumatoid arthritis. N Engl J Med 344: 907-916, 2001.

26. Feldmann M: Development of anti-TNF therapy for rheumatoid arthritis. Nat Rev Immunol 2: 364-371, 2002.

27. Ehrenstein MR, Evans JG, Singh A, Moore S, Warnes G, Isenberg DA and Mauri C: Compromised function of regulatory $\mathrm{T}$ cells in rheumatoid arthritis and reversal by anti-TNFalpha therapy. J Exp Med 200: 277-285, 2004.

28. Hashizume M and Mihara M: The roles of interleukin- 6 in the pathogenesis of rheumatoid arthritis. Arthritis 2011: 765624, 2011.

29. de Benedetti F, Massa M, Robbioni P, Ravelli A, Burgio GR and Martini A: Correlation of serum interleukin-6 levels with joint involvement and thrombocytosis in systemic juvenile rheumatoid arthritis. Arthritis Rheum 34: 1158-1163, 1991.

30. Saftig P and Reiss K: The 'A Disintegrin And Metalloproteases' ADAM10 and ADAM17: Novel drug targets with therapeutic potential? Eur J Cell Biol 90: 527-535, 2011

31. Zhao H, Zhu J, Cui K, Xu X, O'Brien M, Wong KK, Kesari S, Xia W and Wong ST: Bioluminescence imaging reveals inhibition of tumor cell proliferation by Alzheimer's amyloid beta protein. Cancer Cell Int 9: 15, 2009.

32. Murai T, Miyazaki Y, Nishinakamura H, Sugahara KN, Miyauchi T, Sako Y, Yanagida T and Miyasaka M: Engagement of CD44 promotes Rac activation and CD44 cleavage during tumor cell migration. J Biol Chem 279: 4541-4550, 2004.

33. Zhang W, Liu S, Liu K, Ji B, Wang Y and Liu Y: Knockout of ADAM10 enhances sorafenib antitumor activity of hepatocellular carcinoma in vitro and in vivo. Oncol Rep 32: 1913-1922, 2014. 
34. Yuan Q, Cai S, Zhang X, Liu Z, Li Z, Luo X, Xiong C, Wang J, $\mathrm{Hu} \mathrm{J}$ and Ruan J: A new protoapigenone analog RY10-4 induces apoptosis and suppresses invasion through the PI3K/Akt pathway in human breast cancer. Cancer Lett 324: 210-220, 2012.

35. Yuan H, Yang P, Zhou D, Gao W, Qiu Z, Fang F, Ding S and Xiao W: Knockdown of sphingosine kinase 1 inhibits the migration and invasion of human rheumatoid arthritis fibroblast-like synoviocytes by down-regulating the PI3K/AKT activation and MMP-2/9 production in vitro. Mol Biol Rep 41: 5157-5165, 2014.
36. Xue M, McKelvey K, Shen K, Minhas N, March L, Park SY and Jackson CJ: Endogenous MMP-9 and not MMP-2 promotes rheumatoid synovial fibroblast survival, inflammation and cartilage degradation. Rheumatology (Oxford) 53: 2270-2279, 2014

37. Li F, Li X, Kou L, Li Y, Meng F and Ma F: SUMO-conjugating enzyme UBC9 promotes proliferation and migration of fibroblast-like synoviocytes in rheumatoid arthritis. Inflammation 37: 1134-1141, 2014. 